\title{
Static tensioning promotes hamstring tendons force relaxation more reliably than cycling tensioning
}

\author{
Sérgio Rocha Piedade a ${ }^{\mathrm{a}}$ Inácio Maria Dal Fabbro ${ }^{\mathrm{b}}$, Martha Maria Mischan ${ }^{\mathrm{c}}$, \\ Cezar Piedade Jr. ${ }^{\mathrm{d}}$, Nicola Maffulli ${ }^{\mathrm{e}, *}$
}

\author{
a Exercise and Sports Medicine Group, School of Medical Sciences, UNICAMP, Campinas, Brazil Department of Orthopaedic and Traumatology School of Medical Sciences \\ UNICAMP, Campinas, Brazil \\ b School of Agricultural Engineering, UNICAMP, Campinas, Brazil \\ c Department of Biostatistics, UNESP, Botucatu, Brazil \\ d Department of Agricultural Rural Engineering, UNESP, Botucatu, Brazil \\ e Department of Musculoskeletal Disorders, Faculty of Medicine and Surgery, University of Salerno, 84081 Baronissi, Salerno, Italy; Centre for Sports and Exercise Medicine, Barts \\ and The London School of Medicine and Dentistry, Mile End Hospital, 275 Bancroft Road, London E1 4DG, England
}

\section{A R T I C L E I N F O}

\section{Article history:}

Received 22 October 2016

Received in revised form 15 March 2017

Accepted 26 April 2017

\section{Keywords:}

Anterior cruciate ligament

Graft tensioning

Hamstring graft

Sports medicine

Tendon stress-strain

\begin{abstract}
A B S T R A C T
Background: Graft elongation might be a major reason for increased anterior laxity after anterior cruciate ligament (ACL) reconstruction. This study analyzed the force relaxation values and their stabilization when single strands of the gracilis and semitendinosus tendons underwent cyclic and static tensioning at $2.5 \%$ strain level, and compared the efficiency of static and cyclic tensioning in promoting force relaxation.

Methods: Eighteen gracilis tendons and 18 semitendinosus tendons from nine male cadavers (mean age: 22.44 years) were subjected to 10 in vitro cyclic loads at $2.5 \%$ strain level, or to a static load at $2.5 \%$ strain level.

Results: During cyclic loading, the reduction in force values tended to stabilize after the sixth cyclic load, while, in the case of static loading, this stabilization occurred by the second minute. Comparing static and cyclic loading, the gracilis tendon had similar mechanical responses in both conditions, while the semitendinosus tendon showed greater force relaxation in static compared with cyclic loading.

Conclusions: Considering that the semitendinosus tendon is the main component of the hamstring graft, its biomechanical response to loading should guide the tensioning protocol. Therefore, static tensioning seems more effective for promoting force relaxation of the semitendinosus tendon than cyclic tensioning. The gracilis tendon showed a similar mechanical response to either tensioning protocols.
\end{abstract}

(C) 2017 Elsevier B.V. All rights reserved.

\section{Introduction}

The success of surgical reconstruction of the anterior cruciate ligament (ACL) depends on several variables, including: graft selection, bone tunnels positioning, fixation system, postoperative rehabilitation program, and the mechanical behavior of the graft after fixation [1-5]. Graft elongation after definitive fixation might be a major cause of surgical failure [6]. Although tendon

\footnotetext{
* Corresponding author at: Department of Musculoskeletal Disorders, Faculty of Medicine and Surgery, University of Salerno, 84081 Baronissi, Salerno, Italy.

E-mail address: n.maffulli@qmul.ac.uk (N. Maffulli).
} 
grafts preconditioning on the graft board seem to be helpful to prevent post-implantation graft elongation, it is not regularly performed in clinical practice. Therefore, during ACL reconstruction, graft tensioning is commonly applied after one of the ends of the graft has been fixed. This procedure aims to reduce the occurrence of excessive graft elongation postoperatively, and it is obtained by loading the graft statically or cyclically before undertaking definitive fixation. However, the ideal level of graft tensioning when using tendon grafts to reconstruct the ACL remains to be established. Graft tensioning is commonly undertaken, but it is still performed in an empirical fashion, as there is no consensus on the ideal tensioning levels [7,8]. Clinically, undertensioning the graft could lead to the risk of residual laxity after implantation. This must be balanced against the risk of overconstraining the knee, which may lead to pathologic stresses on the articular cartilage, graft failure, or infrapatellar contracture [9,10].

Human tendons have no uniform cross-sectional area. Therefore, calculating the adequate tension to be applied to each tendon graft has clinical limitations; this issue is particularly evident when tendons with different diameters and sizes are used together, as in ACL reconstruction.

The response of tendon grafts subjected to tension has been studied [11,12], but the behavior of the grafts used in ACL reconstruction is complex, as commonly two tendons (gracilis and semitendinosus) are used together to reconstruct the ACL [13]. During normal activities, tendons and ligaments in humans are subjected to strain $<5 \%$, which is the upper physiological limit before plastic deformation takes place $[14,15]$. Knowledge of the response of tendons to tensioning may play a major role in optimizing surgical results.

The present study investigated the behavior of single strands of human gracilis and semitendinosus tendons subjected to in vitro static or cyclic tensioning at $2.5 \%$ strain to define the response to static or cyclic loading of human gracilis and semitendinosus tendons, and the influence of relative deformation and force on controlling graft tension. This study aimed to determine the force relaxation values and its stabilization when single strands of the gracilis and semitendinosus tendons underwent cyclic and static tensioning at $2.5 \%$ strain level. The second aim of the present investigation was to compare the efficiency of static and cyclic tensioning in promoting force relaxation matching the percentage of mean force relaxation values until the second minute and sixth cycle of testing, which is a period where the force relaxation values were stabilized in the respective mechanical tests that were performed.

\section{Materials and methods}

This study was conducted on 18 human gracilis tendons and 18 semitendinosus tendons removed from nine male cadavers (age range: 16-34 years, mean age 22.44 years). All the procedures described in the present investigations were approved by the Medical Research Ethical Committee of the Medical Science School of this University under Project number 168/99.

All the tendons were harvested within $24 \mathrm{~h}$ of death. A longitudinal incision was performed centered on the pes anserinus over the anteromedial aspect of the tibia. The sartorius aponeurosis was opened by sharp dissection, and the insertions of gracilis and semitendinosus tendons on the tibia were visualized. The tendons were isolated and harvested with an open stripper. The tendons were then wrapped in gauze soaked in $0.9 \%$ saline solution and stored in sealed plastic bags at $-20{ }^{\circ} \mathrm{C}$. Before testing, the tendons were gently defrosted at room temperature overnight $(10 \mathrm{~h})$ to conduct the tests [16].

The tendons were divided into two groups according to the mechanical assays: static or cyclic tensioning. The first group of tendons was subjected to 10 in vitro cyclic loads at $2.5 \%$ strain, and the value of the deforming load used for each cycle was recorded. The second group was subjected to a $2.5 \%$ static strain, and the value of the deforming force was recorded at the end of each minute. In this study, the relative deformation level adopted was $2.5 \%$, as it falls within the physiological limit of human tendons. During the tests, the tendons were fixed to a sinusoidal clamp system with grooves. Each clamp had four screws tightened to a torque of $2.5 \mathrm{~N} \mathrm{~mm}$ using an AVT 100A Britool torquemeter model (Britool Ltd, Sheffield, England) with a scale ranging from

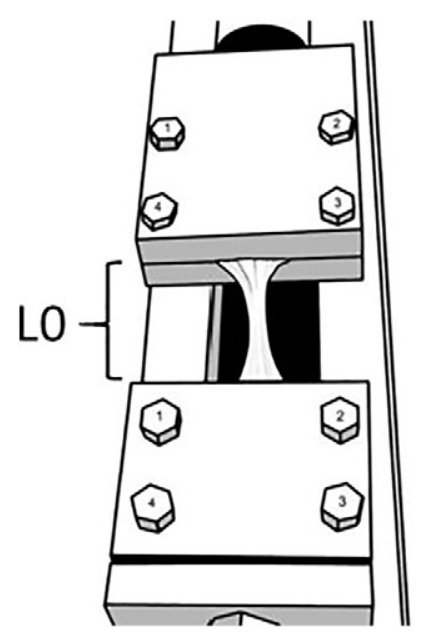

Figure 1. Schematic drawing of the sinusoidal clamp system used in both tensioning and the calculus of initial body test (L0). 
2.5-11 $\mathrm{N} \mathrm{mm}$. The initial length of the test body (LO) was defined as the tendon length between the clamps; a mean length of $31.8 \mathrm{~mm}$ (SD 1.1) was maintained (Figure 1). The tests were carried out using a Lloyd TA 500 press model (Lloyd Instruments Ltd., Fareham, UK). The equipment has a loading capacity of $500 \mathrm{~N}$ and a resolution rate of $0.01 \%$ of load cell rating. The tests were conducted at a loading velocity of $10 \%$ of the $\mathrm{L} 0 / \mathrm{s}$.

A personal computer was used to store all data. The Nexgen 3.0 software (Microsoft, Redmond, WA, USA) was used to analyze the data.

\subsection{Mechanical assays}

\subsubsection{Cyclic tensioning test (10 gracilis and 10 semitendinosus tendons)}

During the cyclic tensioning test, each tendon underwent 10 successive cycles at $2.5 \%$ deformation level. Cyclic tensioning has two phases: tension and relaxation. Once this first level of relative elongation was attained, the relaxation stage began, and the tendon returned to its initial length, which corresponded to the first cycle (Figure 2-A). The force necessary to produce the required deformation was recorded per each cycle.

\subsubsection{Static tensioning test (eight gracilis and eight semitendinosus tendons)}

During the static tensioning test, problems related to the clamp system for fixation invalidated the acquisition of data from two specimens of gracilis and two specimens of semitendinosus. Therefore, eight gracilis tendons and eight semitendinosus tendons were analyzed in the static tensioning test. During the static tensioning test, each tendon underwent $2.5 \%$ relative deformation. When a $2.5 \%$ relative deformation was reached, the tendon was maintained in this condition for 10 min (Figure 2-B). The force values were recorded each minute.

\subsection{Statistical analysis}

The data obtained were statistically analyzed using the SAS System for Windows program (Statistical Analysis System, version 9.2, SAS Institute Inc., 2002-2008, Cary, NC, USA). Confidence intervals were set at 95\%, and significance was set at $\alpha=0.05$. Regression analysis and analysis of variance were used to evaluate the reduction in force values obtained for the semitendinosus and gracilis tendons in both tensioning regimens. The percentage values of the mean force relaxation obtained until the sixth cycle and the second minute at cyclic and static tensioning, respectively, were analyzed. The homogeneity of the variance of the measured variables was verified using the Levene's test. Factorial analysis of variance was performed in a completely randomized design, and the Tukey test was used to test for significance of the average results of the loading regimens.

\section{Results}

Figure 2 shows a typical example of the results of an experiment of cyclic (Figure 2-A) and static (Figure 2-B) tensioning in a tendon.

\subsection{Mean force values and range obtained in both mechanical assays}

Tables 1 and 2 present the mean force values (N), range, standard deviation (SD) and percentage of force relaxation related to the first cycle and the global relaxation obtained during the first, sixth, seventh, eighth and tenth cycles during the cyclic

\section{(2-A) cyclic tensioning (2-B) static tensioning}
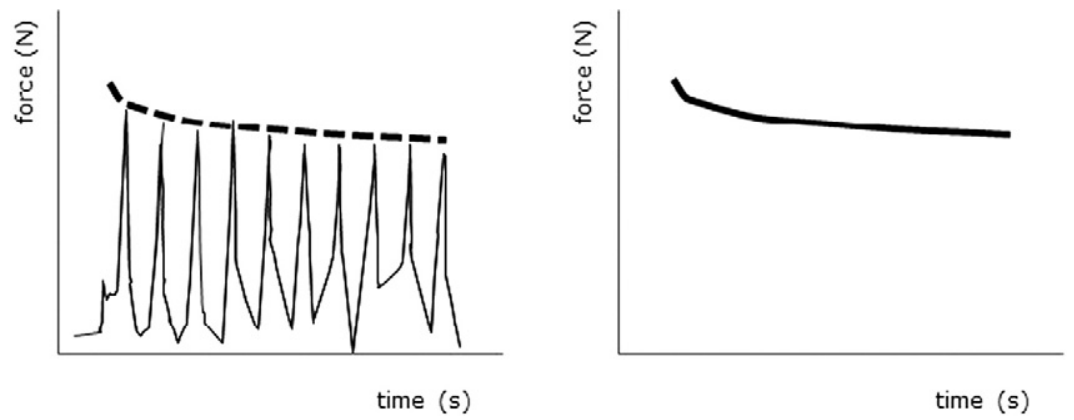

Figure 2. Schematics of static and cyclic tendon tensioning and the resulting force relaxation curves (dotted line, 2-A; solid line, 2-B, respectively) at the 2.5\% strain level. 
Table 1

Mean force values $(\mathrm{N})$, range, SD and percentage of force relaxation related to the first cycle and the global relaxation obtained during the first, sixth, seventh, eighth and tenth cycles for gracilis tendon during cyclic tensioning.

\begin{tabular}{|c|c|c|c|c|c|}
\hline Tendons $(\mathrm{n}=10)$ & 1st cycle & 6th cycle & 7th cycle & 8th cycle & 10th cycle \\
\hline Mean force values & 13.28 & 11.10 & 10.49 & 9.83 & 9.35 \\
\hline Range & $5.29-38.72$ & $3.50-32.10$ & $3.48-29.74$ & $3.41-27.95$ & $3.39-27.38$ \\
\hline (Standard deviation) & $(11.13)$ & $(9.44)$ & $(8.92)$ & $(8.20)$ & $(8.04)$ \\
\hline \multirow{2}{*}{ Percentage of force relaxation related to } & 1st cycle & $17.19 \%$ & $22.00 \%$ & $26.48 \%$ & $31.23 \%$ \\
\hline & Global relaxation & $56.35 \%$ & $71.50 \%$ & $85.61 \%$ & $100 \%$ \\
\hline
\end{tabular}

Table 2

Mean force values (N), range, SD and percentage of force relaxation related to the first cycle and the global relaxation obtained during the first, sixth, seventh, eighth and tenth cycles for semitendinosus tendon during cyclic tensioning.

\begin{tabular}{|c|c|c|c|c|c|}
\hline Tendons $(\mathrm{n}=10)$ & 1st cycle & 6th cycle & 7th cycle & 8th cycle & 10th cycle \\
\hline Mean values & 12.08 & 10.61 & 10.08 & 9.33 & 8.84 \\
\hline $\begin{array}{l}\text { Range } \\
\text { (Standard deviation) }\end{array}$ & $\begin{array}{l}4.10-43.60 \\
(11.90)\end{array}$ & $\begin{array}{l}3.90-37.00 \\
(10.09)\end{array}$ & $\begin{array}{l}3.64-35.91 \\
(9.81)\end{array}$ & $\begin{array}{l}3.14-34.81 \\
(9.49)\end{array}$ & $\begin{array}{l}3.11-34.55 \\
(9.47)\end{array}$ \\
\hline \multirow{2}{*}{ Percentage of force relaxation related to } & 1st cycle & $10.89 \%$ & $15.84 \%$ & $22.39 \%$ & $27.37 \%$ \\
\hline & Global relaxation & $42.20 \%$ & $59.89 \%$ & $82.64 \%$ & $100 \%$ \\
\hline
\end{tabular}

Table 3

Mean force values $(\mathrm{N})$, range, SD and percentage of force relaxation related to the time zero and the global relaxation obtained during the first, second, third and tenth minute for gracilis tendon during static tensioning.

\begin{tabular}{|c|c|c|c|c|c|}
\hline Tendons $(\mathrm{n}=8)$ & Time zero & 1st minute & 2nd minute & 3rd minute & 10th minute \\
\hline Mean values & 7.02 & 6.13 & 5.87 & 5.72 & 5.26 \\
\hline $\begin{array}{l}\text { Range } \\
\text { (Standard deviation) }\end{array}$ & $\begin{array}{l}3.66-13.82 \\
(3.36)\end{array}$ & $\begin{array}{l}2.97-13.37 \\
(3.02)\end{array}$ & $\begin{array}{l}2.75-11.91 \\
(2.92)\end{array}$ & $\begin{array}{l}2.63-11.62 \\
(2.87)\end{array}$ & $\begin{array}{l}2.32-10.88 \\
(2.73)\end{array}$ \\
\hline Percentage of force relaxation related to & $\begin{array}{l}\text { Time zero } \\
\text { Global relaxation }\end{array}$ & $\begin{array}{l}12.91 \% \\
49.57 \%\end{array}$ & $\begin{array}{l}16.82 \% \\
64.62 \%\end{array}$ & $\begin{array}{l}19.17 \% \\
73.71 \%\end{array}$ & $\begin{array}{l}25.95 \% \\
100 \%\end{array}$ \\
\hline
\end{tabular}

tensioning, while Tables 3 and 4 show the mean force values $(\mathrm{N})$, range, SD and percentage of force relaxation related to the time zero and the global relaxation obtained during the first, second, third and tenth minutes for both tendons during the static tensioning.

\subsection{Results of statistical analysis}

Regarding cyclic tensioning, the stabilization of force relaxation values occurred until the sixth cycle for the semitendinosus tendon (force $=\mathrm{f}$ (cycle), $\mathrm{f}=0.0152 \mathrm{c}^{3}-0.2777 \mathrm{c}^{2}+1.0055 \mathrm{c}+11.335, \mathrm{R}^{2}=0.9978$ ) and gracilis tendon (force $=\mathrm{f}(\mathrm{cycle})$, $\left.\mathrm{f}=0.0135 \mathrm{c}^{3}-0.2303 \mathrm{c}^{2}-0.6029 \mathrm{c}+12.895, \mathrm{R}^{2}=0.9996\right)$.

Concerning static tensioning, the semitendinosus tendon showed different force relaxation values on the first and second minute, while tension stabilized by the second minute (force $=\mathrm{f}$ (time), $\mathrm{f}=0.0279 \mathrm{t}^{2}-0.4325 \mathrm{t}+7.5469$, $\mathrm{R}^{2}=0.9017$ )

The gracilis tendon presented similar mechanical behavior, where force relaxation stabilized by the second minute $($ force $=\mathrm{f}$ (time), $\mathrm{f}=0.0266 \mathrm{t}^{2}-0.4112 \mathrm{t}+6.7570, \mathrm{R}^{2}=0.9036$ ).

The semitendinosus tendon showed higher percentage of force relaxation values on static tensioning (19.72) compared with cyclic tensioning (10.89), confirming that static tensioning was more effective in promoting force relaxation (Table 5). On the

Table 4

Mean force values (N), SD and percentage of force relaxation related to the time zero and the global relaxation obtained during the time zero, first, second, third and tenth minute for semitendinosus tendon during static tensioning.

\begin{tabular}{llllll}
\hline Tendons $(\mathrm{n}=8)$ & Time zero & 1st minute & 2nd minute & 3rd minute & 10th minute \\
\hline Mean values & 7.82 & 6.89 & 6.61 & 6.46 & 5.98 \\
Range & $3.08-25.83$ & $2.47-24.19$ & $2.31-23.64$ & $2.21-23.40$ & $1.97-22.31$ \\
(Standard deviation) & $(7.48)$ & $(7.18)$ & $(7.07)$ & $(7.03)$ & $(6.77)$ \\
& & $15.23 \%$ & $19.72 \%$ & $22.25 \%$ & $29.52 \%$ \\
Percentage of force relaxation related to & Time zero & Global relaxation & $50.71 \%$ & $66.06 \%$ & $74.63 \%$ \\
\hline
\end{tabular}


Table 5

Comparison of percentage of force relaxation until the sixth cycle and second minute in both tendons studied.

\begin{tabular}{lll}
\hline Tendon & Tensioning & \\
\cline { 2 - 3 } & Cyclic & Static \\
\hline Semitendinosus & $10.89 \mathrm{~b}$ & $19.72 \mathrm{a}$ \\
Gracilis & 17.19 & 16.82 \\
\hline
\end{tabular}

Small letters compare the horizontal means. Measures with the different letters ( $\mathrm{a}$ and $\mathrm{b}$ ) differ at $\alpha=0.05$ significance level.

other hand, the gracilis tendon demonstrated no difference in percentage of force values in relation to cyclic (17.19) and static (16.82) tensioning regimens (Table 5).

\section{Discussion}

The most important finding of this study was that cyclic and static tendon graft tensioning produce different mechanical responses in the semitendinosus tendon, while the gracilis tendon showed a similar mechanical behavior in either tensioning protocols. This study analyzed an isolated strand of each tendon; nevertheless, the obtained data showed that static tensioning is more effective and reproducible in promoting tendon relaxation in both groups. These observations could be relevant when different tendon graft constructs (double, triple or quadruple strand) with tendons of different cross-sectional areas are employed.

Anterior cruciate ligament $(\mathrm{ACL})$ reconstruction produces $75-85 \%$ good to satisfactory results $[17,18]$. Clinically, postoperative laxity is a sign of failure of ACL reconstruction. Therefore, excluding problems such as graft fixation or tunnel positioning, graft tensioning is a factor to be taken into consideration $[4,7,19]$.

Tendon grafts exhibit viscoelastic behavior characterized by force relaxation or creep phenomena that could lead to decreased graft tensioning and increased knee laxity over time $[11,13,15]$. In this context, graft tensioning is commonly undertaken to minimize the occurrence of these phenomena [16]. Although isolated cyclic or static tensioning is commonly used to promote single graft tensioning, it could be hypothesized that the mechanical response of tensioning protocol (cyclic or static) could vary when tendons with different viscoelastic properties are used together. Therefore, the biomechanical investigation described in the present study provides better understanding of the mechanical response of these two commonly used tendon grafts and may guide the choice of graft tensioning protocols.

Yasuda et al. randomized patients into three groups, applying initial graft tensions of 20,40 , and $80 \mathrm{~N}$ to double-stranded hamstring grafts connected in series with polyester tape [20]. A statistically significant association was found between initial graft tension at the time of fixation and anteroposterior laxity after two years, and the high-tension group had significantly less anterior laxity than the low-tension group (i.e. closer to normal). Nicholas et al. recently reported similar findings with patellar tendon grafts [21]. In contrast, van Kampen et al. and Yoshiya et al. compared initial graft tension levels of 20 and $40 \mathrm{~N}$ and 25 and $50 \mathrm{~N}$, respectively, using patellar tendon grafts [22,23]. They found no significant difference in anteroposterior laxity values between treatment groups two years after the index reconstruction. Andersen and Jorgensen determined that a significant decrease in knee motion occurred when the graft tension was $>33 \mathrm{~N}$ [24]. Boguszewski et al. analyzed, in in vitro laboratory tests, the mechanical response of graft tissue for ACL reconstruction after different preconditioning protocols [25]. The authors subjected four commonly used grafts to a cyclic load of $89 \mathrm{~N}$ and showed no effect of the preconditioning compared to the no preconditioning condition on the anterior knee laxity. Analyzing the tension levels of quadruple-strand hamstring tendon graft preconditioned at $80 \mathrm{~N}$ and $160 \mathrm{~N}$, Elias et al. observed that increasing the tension on the graft board could decrease the postoperative loss of tension given by the viscoelastic behavior of the tendons [26]. Regarding the different force values proposed in these previous studies and the results regarding the mechanical graft tissue response, such as the residual anterior knee laxity after tensioning, it appears that simply applying a given force is not the best way of controlling graft tensioning.

It is believed that there are no guidelines regarding the optimal tension for each tendon graft [27]. In clinical practice, the tension applied to the tendon graft ranges from 20 to $80 \mathrm{~N}$, whereas other authors accomplish graft tensioning "by hand" [5,28,29]. The amount of force needed to tension a graft should be titrated according to the size and diameter of the tendons [30], and should vary when two tendons with different cross sectional areas are used together in the same construct.

The present study analyzed an individual single strand of each tendon. Nevertheless, these data should be taken into account when two different tendons, namely gracilis and semitendinosus, are used together to produce a graft to be used for ACL reconstruction. A single strand of the gracilis and semitendinosus tendons presents different mechanical responses when subjected to uniaxial tensile tests [31,32]: the gracilis tendons were more deformable over time, and showed lower ultimate strength values compared with the semitendinosus tendon.

In the present study, when plotted, force relaxation values produced a curve that showed a similar structure on cyclic (Figure 2-A, solid line) and static (Figure 2-B, dotted line) tensioning, showing that tension stabilized over the time of observation. Nevertheless, the magnitude of its mechanical response was different and specific for each tendon [33]. In addition, in the present study, static tensioning was more effective in promoting force relaxation of the semitendinosus than cyclic tensioning, while the gracilis tendon showed a similar mechanical response in both tensioning protocols. The relative deformation instead of just force values seemed 
more reasonable and efficient in promoting better control of tensioning. These observations could be more relevant when different tendon graft constructs (double, triple or quadruple strand) show different cross section areas.

This study had several limitations. For example, the mechanical assays were not performed immediately after harvesting; the tendons were stored in sealed plastic bags at $-20{ }^{\circ} \mathrm{C}$ and defrosted overnight before performing the mechanical assays. Also, the mechanical behavior of two hamstring tendons (semitendinosus and gracilis) was analyzed, and other potential tendon grafts (such as patellar and quadriceps tendons) were not included in this study. Although this study analyzed an individual single strand tendon, which the authors were aware is different from a typical ACL graft construct (double, triple or quadruple strand), it allowed investigation of the influence of the relative deformation and the force on controlling the tension applied to each tendon. This imparts better understanding of the mechanical response of semitendinosus and gracilis tendon grafts following both tensioning protocols. Also, it should be considered that these data were obtained in in vitro laboratory tests: the results should be translated into clinical practice by appropriately powered well-performed clinically relevant trials.

\section{Conclusion}

Considering that the semitendinosus tendon is the main component of the hamstring graft, its biomechanical response to loading should guide the tensioning protocol. Therefore, static tensioning seems more reasonable and effective for promoting force relaxation of the semitendinosus tendon than cyclic tensioning. The gracilis tendon showed a similar mechanical response to either tensioning protocols.

\section{References}

[1] Macaulay AA, Perfetti DC, Levine WN. Anterior cruciate ligament graft choices. Sports Health 2012;4(1):63-8.

[2] Shaerf DA, Pastides PS, Sarraf KM, Willis-Owen CA. Anterior cruciate ligament reconstruction best practice: a review of graft choice. World J Orthop 2014;18:5(1):23-9.

[3] Magen HE, Howell SM, Hull ML. Structural properties of six tibial fixation methods for anterior cruciate ligament soft tissue grafts. Am J Sports Med 1999;27:35-43.

[4] Steiner EM, Hecker AT, Brown CH, Hayes WC. Anterior cruciate ligament graft fixation. Comparison of hamstring and patellar tendon grafts. Am J Sports Med 1994:22:240-7.

[5] Aglietti P, Buzzi R, Giron F, Simeone AJ, Zaccherotti G. Arthroscopic-assisted anterior cruciate ligament reconstruction with the central third patellar tendon. A 5-8-year follow-up. Knee Surg Sports Traumatol Arthrosc 1997;5(3):138-44.

[6] Boorman RS, Shrive NG, Frank CB. Immobilization increases the vulnerability of rabbit medial collateral ligament autograft to creep. J Orthop Res 1998;16:682-9.

[7] Kirwan GW, Bourke MG, Chipchase L, Dalton PA, Russell TG. Initial graft tension and the effect on postoperative patient functional outcomes in anterior cruciate ligament reconstruction. Arthroscopy 2013;29(5):934-41.

[8] Arneja S, McConkey MO, Mulpuri K, Chin P, Gilbart MK, Regan WD, et al. Systematic review graft tensioning in anterior cruciate ligament reconstruction: a systematic review of randomized controlled trials. Arthroscopy 2009;25(2):200-7.

[9] Markolf KL, O'Neill G, Jackson SR, McAllister DR. Reconstruction of knees with combined cruciate deficiencies. A biomechanical study. J Bone Joint Surg Am 2003; 85(9):1768-74.

[10] Austin JC, Phornphutkul C, Wojtys EM. Loss of knee extension after anterior cruciate ligament reconstruction: effects of knee position and graft tensioning. J Bone Joint Surg Am 2007;89:1565-74.

[11] Sherman SL, Chalmers PN, Yanke AB, Bush-Joseph CA, Verma NN, Cole BJ, et al. Graft tensioning during knee ligament reconstruction: principles and practice. J Am Acad Orthop Surg 2012;20(10):633-45.

[12] Koga H, Muneta T, Yagishita K, Watanabe T, Mochizuki T, Horie M, et al. Effect of initial graft tension on knee stability and graft tension pattern in double-bundle anterior cruciate ligament reconstruction. Arthroscopy 2015;31(9):1756-63.

[13] Conteduca F, Morelli F, Ferretti A. Viscoelastic properties of the semitendinosus and gracilis tendons in the reconstruction of the ACL: an in vivo evaluation. Chir Organi Mov 2003;88(1):75-82.

[14] Abrahams M. Mechanical behavior of tendon in vivo. Med Biol Eng 1967;5:433-43.

[15] Fung YC. The meaning of the constitutive equation. In: Fung YC, editor. Biomechanics-mechanical properties of living tissues. New York: Springer; 1993. p. $23-65$.

[16] Brum J, Bernal M, Gennisson JL, Tanter M. In vivo evaluation of the elastic anisotropy of the human Achilles tendon using shear wave dispersion analysis. Phys Med Biol 2014;59(3):505-23.

[17] Rodríguez-Roiz JM, Caballero M, Ares O, Sastre S, Lozano L, Popescu D. Return to recreational sports activity after anterior cruciate ligament reconstruction: a oneto six-year follow-up study. Arch Orthop Trauma Surg 2015;135(8):1117-22.

[18] Ardern CL, Webster KE, Taylor NF, Feller JA. Return to sport following anterior cruciate ligament reconstruction surgery: a systematic review and meta-analysis of state of play. Br J Sports Med 2011;45(7):596-606.

[19] Dheerendra SK, Khan SW, Singhal R, Shivarathre DG, Pydisetty R, Johnstone D. Anterior cruciate ligament graft choices: a review of current concepts. Open Orthop J 2012;6(Suppl. 2: M4):281-6.

[20] Yasuda K, Tsujino J, Tanabe Y, Kaneda K. Effects of initial graft tension on clinical outcome after anterior cruciate ligament reconstruction: autogenous doubled hamstring tendons connected in series with polyester tapes. Am J Sports Med 1997;25:99-106.

[21] Nicholas SJ, D'Amato MJ, Mullaney MJ, Tyler TF, Kolstad K, McHugh MP. A prospectively randomized double-blind study on the effect of initial graft tension on knee stability after anterior cruciate ligament reconstruction. Am J Sports Med 2004;32:1881-6.

[22] van Kampen A, Wymenga AB, van der Heide HJL. The effect of different graft tensioning in anterior cruciate ligament reconstruction: a prospective randomized study. Arthroscopy 1998; $14: 845-50$.

[23] Yoshiya S, Andrish JT, Manley MT, Bauer TW. Graft tension in anterior cruciate ligament reconstruction: an in vivo study in dogs. Am J Sports Med 1987;15:464-9.

[24] Andersen HN, Jorgensen U. The immediate postoperative kinematic state after anterior cruciate ligament reconstruction with increasing preoperative tension. Knee Surg Sports Traumatol Arthrosc 1998;6:S62-9.

[25] Boguszewski DV, Joshi NB, Wang D, Markolf KL, Petrigliano FA, McAllister DR. Effect of different preconditioning protocols on anterior cruciate ligament reconstruction with four commonly used grafts. J Bone Joint Surg Am 2015;97(13):1059-66.

[26] Elias JJ, Kilambi S, Ciccone II WJ. Tension level during preconditioning influences hamstrings tendon graft properties. Am J Sports Med 2009;37(2):334-8.

[27] Tohyama H, Yasuda K. Significance of graft tension in anterior cruciate ligament reconstruction. Basic background and clinical outcome. Knee Surg Sports Traumatol Arthrosc 1998;6(Suppl. 1):S30-7.

[28] Zarins B, Rowe CR. Combined anterior cruciate ligament reconstruction using semitendinosus tendon and iliotibial tract. J Bone Joint Surg Am 1986;68(2):160-77.

[29] Keays SL, Bullock-Saxton JE, Keays AC, Newcombe PA, Bullock MI. A 6-year follow-up of the effect of graft site on strength, stability, range of motion, function, and joint degeneration after anterior cruciate ligament reconstruction: patellar tendon versus semitendinosus and gracilis tendon graft. Am J Sports Med 2007;35(5): 729-39.

[30] Janssen RP, van der Velden MJ, van den Besselaar M, Reijman M. Prediction of length and diameter of hamstring tendon autografts for knee ligament surgery in Caucasians. Knee Surg Sports Traumatol Arthrosc 2017;25(4):1199-204. 
[31] Pailhé R, Cavaignac E, Murgier J, Laffosse JM, Swider P. Biomechanical study of ACL reconstruction grafts. J Orthop Res 2015;33(8):1188-96.

[32] Piedade SR, Del Fabbro IM, Vidal BC, Gamba R. Uniaxial traction test on human gracilis and semitendinosus tendon. Acta Orthop Bras 2001;9(1):5-11.

[33] Burks RT, Leland R. Determination of graft tension before fixation in anterior cruciate ligament reconstruction. Arthroscopy 1988;4(4):260-6. 